\title{
Polar Codes: Robustness of the Successive Cancellation Decoder with Respect to Quantization
}

\author{
S. Hamed Hassani and Rüdiger Urbanke \\ School of Computer and Communication Sciences, EPFL \\ Email: \{seyedhamed.hassani, rudiger.urbanke\}@epfl.ch
}

\begin{abstract}
Polar codes provably achieve the capacity of a wide array of channels under successive decoding. This assumes infinite precision arithmetic. Given the successive nature of the decoding algorithm, one might worry about the sensitivity of the performance to the precision of the computation.

We show that even very coarsely quantized decoding algorithms lead to excellent performance. More concretely, we show that under successive decoding with an alphabet of cardinality only three, the decoder still has a threshold and this threshold is a sizable fraction of capacity. More generally, we show that if we are willing to transmit at a rate $\delta$ below capacity, then we need only $c \log (1 / \delta)$ bits of precision, where $c$ is a universal constant.
\end{abstract}

\section{INTRODUCTION}

Since the invention of polar codes by Arikan, [1], a large body of work has been done to investigate the pros and cons of polar codes in different practical scenarios (for a partial list see [2]-[9]).

We address one further aspect of polar codes using successive decoding. We ask whether such a coding scheme is robust. More precisely, the standard analysis of polar codes under successive decoding assumes infinite precision arithmetic. Given the successive nature of the decoder, one might worry how well such a scheme performs under a finite precision decoder. A priori it is not clear whether such a coding scheme still shows any threshold behavior and, even if it does, how the threshold scales in the number of bits of the decoder.

We show that in fact polar coding is extremely robust with respect to the quantization of the decoder. In Figure 1 we show the achievable rate using a simple successive decoder with only three messages, called the decoder with erasures, when transmission takes place over several important channel families. As one can see from this figure, in particular for channels with high capacity, the fraction of the capacity that is achieved by this simple decoder is close to 1 , i.e., even this extremely simple decoder almost achieves capacity. We further show that, more generally, if we want to achieve a rate which is $\delta$ below capacity by $\delta>0$, then we need at most $c \log (1 / \delta)$ bits of precision (all the logarithms in this paper are in base 2).

The significance of our observations goes beyond the pure computational complexity which is required. Typically, the main bottleneck in the implementation of large high speed coding systems is memory. Therefore, if one can find decoders which work with only a few bits per message then

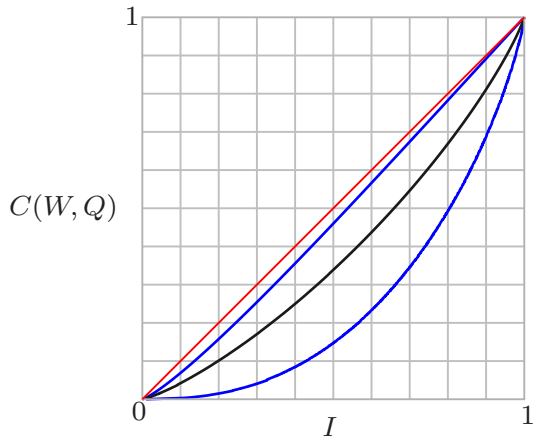

Fig. 1. The maximum achievable rate, call it $C(W, Q)$, of a simple three message decoder, called the decoder with erasures, as a function of the capacity of the channel for different channel families. From top to bottom: the first curve corresponds to the family of binary erasure channels (BEC) where the decoder with erasures is equivalent to the original SC decoder and, hence, the maximum achievable rate is the capacity itself. The second curve corresponds to the family of binary symmetric channels (BSC). The third curve corresponds to the family of binary additive white Gaussian channels (BAWGN). The curve at the bottom corresponds to a universal lower bound on the achievable rate by the decoder with erasures.

this can make the difference whether a coding scheme is implementable or not.

\section{A. Basic setting and definitions}

Let $W: \mathcal{X} \rightarrow \mathcal{Y}$ be a binary memoryless symmetric (BMS) channel, with input alphabet $\mathcal{X}=\{0,1\}$, output alphabet $\mathcal{Y}$, and the transition probabilities $\{W(y \mid x): x \in \mathcal{X}, y \in \mathcal{Y}\}$. Also, let $I(W)$ denote the capacity of $W$.

Let $G_{2}=\left[\begin{array}{ll}1 & 0 \\ 1 & 1\end{array}\right]$. The generator matrix of polar codes is defined through the Kronecker powers of $G_{2}$, denoted by $G_{N}=G_{2}^{\otimes n}$. Throughout the paper, the variables $N$ and $n$ are related as $N=2^{n}$. Let us review very briefly how the generator matrix of polar codes is constructed. Consider the $N \times N$ matrix $G_{N}$ and let us label the rows of the matrix $G_{N}$ from top to bottom by $0,1, \cdots, N-1$. Now assume that we desire to transmit binary data over the channel $W$ at rate $R<I(W)$ with block-length $N$. One way to accomplish this is to choose a subset $\mathcal{I} \subseteq\{0, \cdots, N-1\}$ of size $N R$ and to construct a vector $U_{0}^{N-1}=\left(U_{0}, \cdots, U_{N-1}\right)$ in a way that it contains our $N R$ bits of data at positions in $\mathcal{I}$ and contains, at positions not in $\mathcal{I}$, some fixed value (for example 0 ) which is known to both the encoder and decoder. We then send the codeword $X_{0}^{N-1}=U_{0}^{N-1} G_{N}$ through the channel $W$. We refer to the set $\mathcal{I}$ as the set of chosen indices or information 
indices and the set $\mathcal{I}^{c}$ is called the set of frozen indices. We explain in Section $\amalg-\mathrm{A}$ how the good indices are chosen. At the decoder, the bits $u_{0}, \cdots, u_{N-1}$ are decoded one by one. That is, the bit $u_{i}$ is decoded after $u_{0}, \cdots u_{i-1}$. If $i$ is a frozen index, its value is known to the decoder. If not, the decoder estimates the value of $u_{i}$ by using the output $y_{0}^{N-1}$ and the estimates of $u_{0}, \cdots, u_{i-1}$.

\section{B. Quantized SC decoder}

Let $\mathbb{R}^{*}=\mathbb{R} \cup\{ \pm \infty\}$ and consider a function $Q(x)$ : $\mathbb{R}^{*} \rightarrow \mathbb{R}^{*}$ that is anti-symmetric (i.e., $Q(x)=-Q(-x)$ ). We define the $Q$-quantized SC decoder as a version of the SC decoder in which the function $Q$ is applied to the output of any computation that the SC decoder does. We denote such a decoder by $\operatorname{SCD}_{Q}$.

Typically, the purpose of the function $Q$ is to model the case where we only have finite precision in our computations perhaps due to limited available memory or due to other hardware limitations. Hence, the computations are correct within a certain level of accuracy which the function $Q$ models. Thus, let us assume that the range of $Q$ is a finite set $\mathcal{Q}$ with cardinality $|\mathcal{Q}|$. As a result, all the messages passed through the decoder $\operatorname{SCD}_{Q}$ belong to the set $\mathcal{Q}$.

In this paper we consider a simple choice of the function $Q$ that is specified by two parameters: The distance between levels $\Delta$, and truncation threshold $M$. Given a specific choice of $M$ and $\Delta$, we define $Q$ as follows:

$$
Q(x)=\left\{\begin{array}{lr}
\left\lfloor\frac{x}{\Delta}+\frac{1}{2}\right\rfloor \Delta, & x \in[-M, M], \\
\operatorname{sign}(x) M, & \text { otherwise. }
\end{array}\right.
$$

Note here that $|\mathcal{Q}|=1+\frac{2 M}{\Delta}$.

\section{Summary of results}

Theorem 1 (Main Statement): Consider transmission over a BMS channel $W$ of capacity $I(W)$ using polar codes and a $\operatorname{SCD}_{Q}$ with message alphabet $Q$. Let $C(W, Q)$ denote the maximum rate at which reliable transmission is possible for this setup.

(i) Let $|\mathcal{Q}|=3$. Then there exists a computable decreasing sequence $\left\{U_{n}\right\}_{n \in \mathbb{N}}$ (see (19)) and a computable increasing sequence $\left\{L_{n}\right\}_{n \in \mathbb{N}}\left(\right.$ see $(20)$, so that $L_{n} \leq$ $C(W, Q) \leq U_{n}$ and

$$
\lim _{n \rightarrow \infty} L_{n}=\lim _{n \rightarrow \infty} U_{n} .
$$

In other words, $U_{n}$ is an upper bound and $L_{n}$ is a lower bound on the maximum achievable rate $C(W, Q)$ and for increasing $n$ these two bounds converge to $C(W, Q)$.

(ii) To achieve an additive gap $\delta>0$ to capacity $I(W)$, it suffices to choose $\log |\mathcal{Q}|=c \log (1 / \delta)$.

Discussion: In Figure 1 the value of $C(W, Q),|\mathcal{Q}|=3$, is plotted as a function of $I(W)$ for different channel families (for more details see Section II-D2). A universal lower bound for the maximum achievable rate is also given in Figure 1 This suggests that even for small values of $|\mathcal{Q}|$ polar codes are very robust to quantization. In particular for channels with capacity close to 1 , very little is lost by quantizing. The methods used here are extendable to other quantized decoders.

The rest of the paper is devoted to proving the first part of Theorem 1. Due to space limitation, we have omitted the proof of the second part of theorem 1 as well as the proofs of the lemmas stated in the sequel and we refer the reader to [10] for more details.

\section{General Framework FOR the AnAlysis}

\section{A. Equivalent tree channel model and analysis of the proba- bility of error for the original SC decoder}

Since we are dealing with a linear code, a symmetric channel and symmetric decoders throughout this paper, without loss of generality we confine ourselves to the all-zero codeword (i.e., we assume that all the $u_{i}$ 's are equal to 0 ). In order to better visualize the decoding process, the following definition is handy.

Definition 2 (Tree Channels of Height $n$ ): For each $i \in$ $\{0,1, \cdots, N-1\}$, we introduce the notion of the $i$-th tree channel of height $n$ which is denoted by $T(i)$. Let $b_{1} \ldots b_{n}$ be the $n$-bit binary expansion of $i$. E.g., we have for $n=3$, $0=000,1=001, \ldots, 7=111$. With a slight abuse of notation we use $i$ and $b_{1} \cdots b_{n}$ interchangeably. Note that for our purpose it is slightly more convenient to denote the least (most) significant bit as $b_{n}\left(b_{1}\right)$. Each tree channel consists of $n+1$ levels, namely $0, \ldots, n$. It is a complete binary tree. The root is at level $n$. At level $j$ we have $2^{n-j}$ nodes. For $1 \leq j \leq n$, if $b_{j}=0$ then all nodes on level $j$ are check nodes; if $b_{j}=1$ then all nodes on level $j$ are variable nodes. Finally, we give a label for each node in the tree $T(i)$ : For each level $j$, we label the $2^{n-j}$ nodes at this level respectively from left to right by $(j, 0),(j, 1), \cdots,\left(j, 2^{n-j}-1\right)$.

All nodes at level 0 correspond to independent observations of the output of the channel $W$, assuming that the input is 0 .

An example for $T(3)$ (that is $n=3, b=011$ and $i=3$ ) is shown in Fig. 2 .

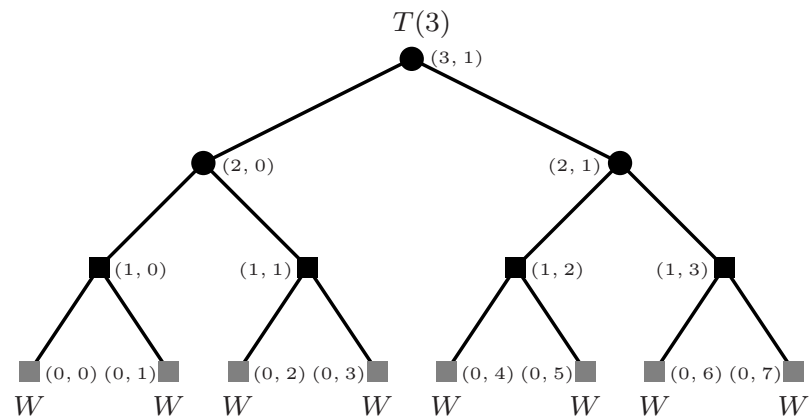

Fig. 2. Tree representation of the tree-channel $T(3)$. The 3-bit binary expansion of 3 is $b_{1} b_{2} b_{3}=011$ (note that $b_{1}$ is the most significant bit). The pair beside each node is the label assigned to it.

Given the channel output vector $y_{0}^{N-1}$ and assuming that the values of the bits prior to $u_{i}$ are given, i.e., $u_{0}=0, \cdots, u_{i-1}=0$, we now compute the probabilities $p\left(y_{0}^{N-1}, u_{0}^{i-1} \mid u_{i}=0\right)$ and $p\left(y_{0}^{N-1}, u_{0}^{i-1} \mid u_{i}=1\right)$ via a simple message passing procedure on the equivalent tree 
channel $T(i)$. We attach to each node in $T(i)$ with label $(j, k)$ a message $11 m_{j, k}$ and we update the messages as we go up towards the root node. We start with initializing the messages at the leaf nodes of $T(i)$. For this purpose, it is convenient to represent the channel in the log-likelihood domain; i.e., for the node with label $(0, k)$ at the bottom of the tree which corresponds to an independent realization of $W$, we plug in the $\log$-likelihood ratio (llr) $\log \left(\frac{W\left(y_{k} \mid 0\right)}{W\left(y_{k} \mid 1\right)}\right)$ as the initial message $m_{0, k}$. That is,

$$
m_{0, k}=\log \left(\frac{W\left(y_{k} \mid 0\right)}{W\left(y_{k} \mid 1\right)}\right) .
$$

Next, the SC decoder recursively computes the messages (llr's) at each level via the following operations: If the nodes at level $j$ are variable nodes (i.e., $b_{j}=1$ ), we have

$$
m_{j, k}=m_{j-1,2 k}+m_{j-1,2 k+1},
$$

and if the nodes at level $j$ are check nodes (i.e., $b_{j}=0$ ), the message that is passed up is

$$
m_{j, k}=2 \tanh ^{-1}\left(\tanh \left(\frac{m_{j-1,2 k}}{2}\right) \tanh \left(\frac{m_{j-1,2 k+1}}{2}\right)\right) .
$$

In this way, it can be shown that ([1]) the message that we obtain at the root node is precisely the value

$$
m_{n, 0}=\log \left(\frac{p\left(y_{0}^{N-1}, u_{0}^{i-1} \mid u_{i}=0\right)}{p\left(y_{0}^{N-1}, u_{0}^{i-1} \mid u_{i}=1\right)}\right) .
$$

Now, given $\left(y_{0}^{N-1}, u_{0}^{i-1}\right)$, the value of $u_{i}$ is estimated as follows. If $m_{n, 0}>0$ we let $u_{i}=0$. If $m_{n, 0}<0$ we let $u_{i}=1$. Finally, if $m_{n, 0}=0$ we choose the value of $u_{i}$ to be either 0 or 1 with probability $\frac{1}{2}$. Thus, denoting $E_{i}$ as the event that we make an error on the $i$-th bit within the above setting, we obtain

$$
\operatorname{Pr}\left(E_{i}\right)=\operatorname{Pr}\left(m_{n, 0}<0\right)+\frac{1}{2} \operatorname{Pr}\left(m_{n, 0}=0\right) .
$$

Given the description of $m_{n, 0}$ in terms of a tree channel, it is now clear that we can use density evolution [2] to compute the probability density function of $m_{n, 0}$. In this regard, at each level $j$, the random variables $m_{j, k}$ are i.i.d. for $k \in$ $\left\{0,1, \cdots, 2^{n-j}-1\right\}$. The distribution of the leaf messages $m_{0, k}$ is the distribution of the variable $\log \left(\frac{W(Y \mid 0)}{W(Y \mid 1)}\right)$, where $Y \sim W(y \mid 0)$. One can recursively compute the distribution of $m_{j, k}$ in terms of the distribution of $m_{j-1,2 k}, m_{j-1,2 k+1}$ and the type of the nodes at level $j$ (variable or check) by using the relations (3), (4) with the fact that the random variables $m_{j-1,2 k}$ and $m_{j-1,2 k+1}$ are i.i.d.

\section{B. Quantized density evolution}

Let us now analyze the density evolution procedure for the quantized decoder. For each label $(j, k)$ in $T(i)$, let $\hat{m}_{j, k}$ represent the messages at this label. The messages $\hat{m}_{j, k}$ take their values in the discrete set $\mathcal{Q}$ (range of the function $Q$ ). It is now easy to see that for the decoder $\operatorname{SCD}_{Q}$ the messages

\footnotetext{
${ }^{1}$ To simplify notation, we drop the dependency of the messages $m_{j, k}$ to the position $i$ whenever it is clear from the context.
}

evolve via the following relations. At the leaf nodes of the tree we plug in the message $\hat{m}_{0, k}=Q\left(\log \left(\frac{W\left(y_{k} \mid 0\right)}{W\left(y_{k} \mid 1\right)}\right)\right)$, and the update equation for $\hat{m}_{(j, k)}$ is

$$
\hat{m}_{j, k}=Q\left(\hat{m}_{j-1,2 k}+\hat{m}_{j-1,2 k+1}\right),
$$

if the node $(j, k)$ is a variable node and

$$
\hat{m}_{j, k}=Q\left(2 \tanh ^{-1}\left(\tanh \left(\frac{\hat{m}_{j-1,2 k}}{2}\right) \tanh \left(\frac{\hat{m}_{j-1,2 k+1}}{2}\right)\right)\right),
$$

if the node $(j, k)$ is a check node. One can use the density evolution procedure to recursively obtain the densities of the messages $\hat{m}_{j, k}$.

Finally, let $\hat{E}_{i}$ denote the event that we make an error in decoding the $i$-th bit, with a further assumption that we have correctly decoded the previous bits $u_{0}, \cdots, u_{i-1}$. In a similar way as in the analysis of the original SC decoder, we get

$$
\operatorname{Pr}\left(\hat{E}_{i}\right)=\operatorname{Pr}\left(\hat{m}_{n, 0}<0\right)+\frac{1}{2} \operatorname{Pr}\left(\hat{m}_{n, 0}=0\right) .
$$

Hence, one way to choose the information bits for the algorithm $\mathrm{SCD}_{Q}$ is to choose the bits $u_{i}$ according to the least values of $\operatorname{Pr}\left(\hat{E}_{i}\right)$.

An important point to note here is that with the decoder $\mathrm{SCD}_{Q}$, the distribution of the messages in the trees $T(i)$ is different than the corresponding ones that result from the original SC decoder. Hence, the choice of the information indices is also specified by the choice of the function $Q$ as well as the channel $W$.

Note here that, since all of the densities takes their value in the finite alphabet $\mathcal{Q}$, the construction of such polar codes can be efficiently done in time $O\left(|\mathcal{Q}|^{2} N \log N\right)$. We refer the reader to [1] for more details.

\section{Gallager Algorithm}

Since our aim is to show that polar codes under successive decoding are robust against quantization, let us investigate an extreme case. The perhaps simplest message-passing type decoder one can envision is the Gallager algorithm. It works with single-bit messages. Does this simple decoder have a nonzero threshold? Unfortunately it does not, and this is easy to see. We start with the equivalent tree-channel model. Consider an arbitrary tree-channel $T(i)$. Since messages are only a single bit, the "state" of the decoder at level $j$ of $T(i)$ can be described by a single non-negative number, namely the probability that the message at level $j$ is incorrect. It is an easy exercise to show that at a level with check nodes the state becomes worse and at a level with variable nodes the state stays unchanged and hence no progress in the decoding is achieved, irrespective of the given tree. In other words, this decoder has a threshold of zero. The problem is the processing at the variable nodes since no progress is achieved there. But since we only have two possible incoming messages there is not much degree of freedom in the processing rules. 


\section{1-Bit Decoder with Erasures}

Motivated by the previous example, let us now add one message to the alphabet of the Gallager decoder, i.e., we also add the possibility of having erasures. In this case $Q(x)$ becomes the sign function, i.e.,

$$
Q(x)=\left\{\begin{array}{cc}
\infty, & x>0 \\
0, & x=0 \\
-\infty, & x<0
\end{array}\right.
$$

As a result, all messages passed by the algorithm $\operatorname{SCD}_{Q}$ take on only three possible values: $\{-\infty, 0, \infty\}$. In this regard, the decoding procedure takes a very simple form. The algorithm starts by quantizing the channel output to one of the three values in the set $\mathcal{Q}=\{-\infty, 0, \infty\}$. At a check node we take the product of the signs of the incoming messages and at a variable node we have the natural addition rule $(0 \leftarrow \infty+-\infty, 0 \leftarrow 0+0$ and $\infty \leftarrow \infty+\infty, \infty \leftarrow \infty+0$ and $-\infty \leftarrow-\infty+-\infty,-\infty \leftarrow-\infty+0)$. Note that on the binary erasure channel, this algorithm is equivalent to the original SC decoder.

Our objective is now to compute the maximum reliable rate that the decoder $\operatorname{SCD}_{Q}$ can achieve for a BMS channel $W$. We denote this quantity by $C(W, Q)$. The analysis is done in three steps:

1) The density evolution procedure: To analyze the performance of this algorithm, first note that since all our messages take their values in the set $\mathcal{Q}$, then all the random variables that we consider have the following form

$$
D=\left\{\begin{array}{cc}
\infty, & \text { w.p. } p, \\
0, & \text { w.p. } e, \\
-\infty, & \text { w.p. } m .
\end{array}\right.
$$

Here, the numbers $p, e, m$ are probability values and $p+e+$ $m=1$. Let us now see how the density evolves through the tree-channels. For this purpose, one should trace the output distribution of (7) and (8) when the input messages are two i.i.d. copies of a r.v. $D$ with pdf as in (11).

Lemma 3: Given two i.i.d. versions of a r.v. $D$ with distribution as in (11), the output of a variable node operation (7), denoted by $D^{+}$, has the following form

$$
D^{+}=\left\{\begin{array}{lr}
\infty, & \text { w.p. } p^{2}+2 p e \\
0, & \text { w.p. } e^{2}+2 p m \\
-\infty, & \text { w.p. } m^{2}+2 e m
\end{array}\right.
$$

Also, the check node operation $(8)$, yields $D^{-}$as

$$
D^{-}=\left\{\begin{array}{cr}
\infty, & \text { w.p. } p^{2}+m^{2}, \\
0, & \text { w.p. } 1-(1-e)^{2}, \\
-\infty, & \text { w.p. } 2 p m .
\end{array}\right.
$$

In order to compute the distribution of the messages $\hat{m}_{n, 0}$ at a given level $n$, we use the method of [1] and define the polarization process $D_{n}$ as follows. Consider the random variable $L(Y)=\log \left(\frac{W(Y \mid 0)}{W(Y \mid 1)}\right)$, where $Y \sim W(y \mid 0)$. The

\footnotetext{
${ }^{2}$ Note here that we have further assumed that $M=\Delta$ and $\Delta \rightarrow 0$.
}

stochastic process $D_{n}$ starts from the r.v. $D_{0}=Q(L(Y))$ defined as

$$
D_{0}= \begin{cases}\infty, & \text { w.p. } p=\operatorname{Pr}(L(Y)>0), \\ 0, & \text { w.p. } e=\operatorname{Pr}(L(Y)=0), \\ -\infty, & \text { w.p. } m=\operatorname{Pr}(L(Y)<0),\end{cases}
$$

and for $n \geq 0$

$$
D_{n+1}= \begin{cases}D_{n}^{+}, & \text {w.p. } \frac{1}{2}, \\ D_{n}^{-}, & \text {w.p. } \frac{1}{2},\end{cases}
$$

where the plus and minus operations are given in (12), 13.

2) Analysis of the process $D_{n}$ : Note that the output of process $D_{n}$ is itself a random variable of the form given in (11). Hence, we can equivalently represent the process $D_{n}$ with a triple $\left(m_{n}, e_{n}, p_{n}\right)$, where the coupled processes $m_{n}, e_{n}$ and $p_{n}$ are evolved using the relations (12) and (13) and we always have $m_{n}+e_{n}+p_{n}=1$. Following along the same lines as the analysis of the original SC decoder in [1], we first claim that as $n$ grows large, the process $D_{n}$ will become polarized, i.e., the output of the process $D_{n}$ will almost surely be a completely noiseless or a completely erasure channel.

Lemma 4: The random sequence $\left\{D_{n}=\left(p_{n}, e_{n}, m_{n}\right), n \geq\right.$ $0\}$ converges almost surely to a random variable $D_{\infty}$ such that $D_{\infty}$ takes its value in the set $\{(1,0,0),(0,1,0)\}$.

We now aim to compute the value of $C(W, Q)=\operatorname{Pr}\left(D_{\infty}=\right.$ $(1,0,0))$, i.e., the highest rate that we can achieve with the 1-Bit Decoder with Erasures. In this regard, a convenient approach is to find a function $f: \mathcal{D} \rightarrow \mathbb{R}$ such that $f((0,1,0))=0$ and $f(1,0,0)=1$ and for any $D \in \mathcal{D}$

$$
\frac{1}{2}\left(f\left(D^{+}\right)+f\left(D^{-}\right)\right)=f(D) .
$$

With such a function $f$, the process $\left\{f\left(D_{n}\right)\right\}_{n \geq 0}$ is a martingale and consequently we have $\operatorname{Pr}\left(D_{\infty}=(1,0,0)\right)=f\left(D_{0}\right)$. Therefore, by computing the deterministic quantity $f\left(D_{0}\right)$ we obtain the value of $C(W, Q)$. However, finding a closed form for such a function seems to be a difficult task Instead, the idea is to look for alternative functions, denoted by $g: \mathcal{D} \rightarrow \mathbb{R}$, such that the process $g\left(D_{n}\right)$ is a super-martingale (submartingale) and hence we can get a sequence of upper (lower) bounds on the value of $\operatorname{Pr}\left(D_{\infty}=(1,0,0)\right)$ as follows. Assume we have a function $g: \mathcal{D} \rightarrow \mathbb{R}$ such that $g((0,1,0))=0$ and $g(1,0,0)=1$ and for any $D \in \mathcal{D}$,

$$
\frac{1}{2}\left(g\left(D^{+}\right)+g\left(D^{-}\right)\right) \leq g(D) .
$$

Then, the process $\left\{g\left(D_{n}\right)\right\}_{n \geq 0}$ is a super-martingale and for $n \geq 0$ we have

$$
\operatorname{Pr}\left(D_{\infty}=(1,0,0)\right) \leq \mathbb{E}\left[g\left(D_{n}\right)\right] .
$$

The quantity $\mathbb{E}\left[g\left(D_{n}\right)\right]$ decreases by $n$ and by using Lemma 4 we have

$$
\operatorname{Pr}\left(D_{\infty}=(1,0,0)\right)=\lim _{n \rightarrow \infty} \mathbb{E}\left[g\left(D_{n}\right)\right]
$$

\footnotetext{
${ }^{3}$ The function $f$ clearly exists as one trivial candidate for it is $f(D)=$ $\operatorname{Pr}\left(D_{\infty}=(1,0,0)\right)$, where $D_{\infty}$ is the limiting r.v. that the process $\left\{D_{n}\right\}_{n \geq 0}$ with starting value $D_{0}=D$ converges to.
} 
In a similar way, on can search for a function $h: \mathcal{D} \rightarrow \mathbb{R}$ such that for $h$ with the same properties as $g$ except that the inequality (16) holds in opposite direction and in a similar way this leads us to computable lower bounds on $C(W, Q)$. It remain to find some suitable candidates for $g$ and $h$. Let us first note that a density $D$ as in 11 can be equivalently represented as a simple BMS channel given in Fig. 3. This equivalence

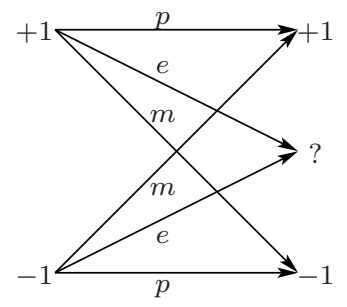

Fig. 3. The equivalent channel for the density $D$ given in 11 .

stems from the fact that for such a channel, conditioned on the event that the symbol +1 has been sent, the distribution of the output is precisely $D$. With a slight abuse of notation, we also denote the corresponding BMS channel by $D$. In particular, it is an easy exercise to show that the capacity $(I(D))$, the Bhattacharyya parameter $(Z(D))$ and the error probability $(E(D))$ of the density $D$ are given as

$$
\begin{aligned}
& I(D)=(m+p)\left(1-h_{2}\left(\frac{p}{p+m}\right)\right), \\
& Z(D)=2 \sqrt{m p}+e, E(D)=1-p-\frac{e}{2},
\end{aligned}
$$

where $h_{2}(\cdot)$ denotes the binary entropy function. Since the function $Q$ is a not an injective function, we have $\frac{I\left(D^{+}\right)+I\left(D^{-}\right)}{2} \leq I(D)$. This implies that the process $I_{n}=$ $I\left(D_{n}\right)^{2}$ is a bounded supermartingale. Furthermore, since $I(D=(1,0,0))=1$ and $I(D=(0,1,0))=0$, we deduce from Lemma 4 that $I_{n}$ converges a.s. to a $0-1$ valued r.v. $I_{\infty}$ and hence

$$
C(W, Q)=\operatorname{Pr}\left(D_{\infty}=(1,0,0)\right)=\operatorname{Pr}\left(I_{\infty}=1\right)=\mathbb{E}\left(I_{\infty}\right) .
$$

Now, from the fact that $I_{n}$ is a supermartingale, we obtain

$$
C(W, Q) \leq \mathbb{E}\left[I_{n}\right] \triangleq U_{n},
$$

for $n \in \mathbb{N}$. In a similar way, one can obtain a sequence of lower bounds for $C(W, Q)$.

Lemma 5: Define the function $F(D)$ as $F(D)=p-4 \sqrt{p m}$ for $D \in \mathcal{D}$. We have $F(D=(1,0,0))=1, F(D=$ $(0,1,0))=0$ and $\frac{F\left(D^{+}\right)+F\left(D^{-}\right)}{2} \geq F(D)$.

Hence, the process $F_{n}=F\left(D_{n}\right)$ is a submartingale and for $n \in \mathbb{N}$ we have

$$
C(W, Q) \geq \mathbb{E}\left[F_{n}\right] \triangleq L_{n} .
$$

Given a BMS channel $W$, one can numerically compute $C(W, Q)$ with arbitrary accuracy using the sequences $L_{n}$ and $U_{n}$ (see Figure 1). Also, for a channel $W$ with capacity $I(W)$ and error probability $E(W)$, we have

$$
E(W) \leq \frac{1-I(W)}{2} .
$$

Therefore, $\inf _{\left\{D: E(D)=\frac{1-I(W)}{2}\right\}} C(D, Q) \leq C(W, Q)$, which leads to the universal lower bound obtained in Figure 1

Example 6: Let the channel $W$ be a BSC channel with cross over probability $\epsilon=0.11$ (hence $I(W) \approx 0.5$ ). Using (22) we obtain

$$
D_{0}=\left\{\begin{array}{lr}
\infty, & \text { w.p. } 1-\epsilon=0.89, \\
-\infty, & \text { w.p. } \epsilon=0.11 .
\end{array}\right.
$$

Therefore, we get $L_{0}=F\left(D_{0}\right)=-0.361$ and $U_{0}=I\left(D_{0}\right)=$ 0.5. We can also compute $L_{1}=\frac{F\left(D_{0}^{+}\right)+F\left(D_{0}^{-}\right)}{2}=-0.191$, $U_{1}=\frac{I\left(D_{0}^{+}\right)+I\left(D_{0}^{-}\right)}{2}=.5$ and

$L_{2}=\frac{F\left(D_{0}^{++}\right)+F\left(D_{0}^{+-}\right)+F\left(D_{0}^{-+}\right)+F\left(D_{0}^{--}\right)}{4}=-0.075$,

$U_{2}=\frac{I\left(D_{0}^{++}\right)+I\left(D_{0}^{+-}\right)+I\left(D_{0}^{-+}\right)+I\left(D_{0}^{--}\right)}{4}=0.498$.

Continuing this way, one can find $L_{10}=0.264, U_{10}=0.474$ and $L_{20}=0.398, U_{20}=0.465$ and so on.

3) Scaling behavior and error exponent: In the last step, we need to show that for rates below $C(W, Q)$ the block-error probability decays to 0 for large block-lengths.

Lemma 7: Let $D \in \mathcal{D}$. We have

$$
Z\left(D^{-}\right) \leq 2 Z(D) \text { and } Z\left(D^{+}\right) \leq 2(Z(D))^{\frac{3}{2}} .
$$

Hence, for transmission rate $R<C(W, Q)$ and block-length $N=2^{n}$, the probability of error of $\operatorname{SCD}_{Q}$, denoted by $P_{e, Q}(N, R)$ satisfies $P_{e, Q}(N, R)=o\left(2^{-N^{\beta}}\right)$ for $\beta<\frac{\log \frac{3}{2}}{2}$.

\section{ACKNOWLEDGMENT}

The authors wish to thank anonymous reviewers for their valuable comments on an earlier version of this manuscript. The work of Hamed Hassani was supported by Swiss National Science Foundation Grant no 200021-121903.

\section{REFERENCES}

[1] E. Arıkan, "Channel polarization: A method for constructing capacityachieving codes for symmetric binary-input memoryless channels," IEEE Trans. Info. Theory, vol. 55, no. 7, pp. 3051-3073, Jul. 2009.

[2] R. Mori and T. Tanaka, "Performance and construction of polar codes on symmetric binary-input memoryless channels," in Proc. ISIT 2009, Seoul, South Korea, pp. 1496-1500, 2009.

[3] I. Tal and A. Vardy, "How to construct polar codes," [online] Available: arXiv:1105.6164v1 [CS.IT].

[4] C. Leroux, I. Tal, A. Vardy and W. J. Gross, "Hardware architectures for successive cancellation decoding of polar codes," in Proc. ICASSP 2011, Prague, Czech Republic, pp. 1665-1668, 2011.

[5] R. Pedarsani, S. H. Hassani, I. Tal and E. Telatar, "On the construction of polar codes," in Proc. ISIT 2011, St. Petersberg, Russia, pp. 11-15, 2011.

[6] I. Tal and A. Vardy, "List decoding of polar codes," in Proc. ISIT 2011, St. Petersberg, Russia, pp. 1-5, 2011.

[7] S. B. Korada, A. Montanari, E. Telatar and R. Urbanke, "An empirical scaling law for polar codes," in Proc. ISIT 2010, Austin, Texas, USA, pp. 884-888, 2010.

[8] S. H. Hassani, K. Alishahi and R. Urbanke, "On the scaling of polar codes: II. The behavior of un-polarized channels," in Proc. ISIT 2010, Austin, Texas, USA, pp. 879-883, 2010.

[9] S. H. Hassani, S. B. Korada and R. Urbanke, "The compound capacity of polar codes," in Proc. 47th Annual Allerton Conference on Communication, Control, and Computing, pp. 16-21, 2009.

[10] S. H. Hassani and R. Urbanke, "Polar Codes: Robustness of the Successive Cancellation Decoder with Respect to Quantization," In preparation. 